\title{
Tracking Substance Abusers in Longitudinal Research: Understanding Follow-Up Contact Difficulty $^{1}$
}

\author{
Maureen A. Walton ${ }^{2}$ \\ University of Michigan Alcohol Research Center

\section{Chathapuram S. Ramanathan} \\ Social Work Consultant Private Practice
}

Thomas M. Reischl

Michigan State University

Studies examining follow-up contact difficulty provide useful information for planning longitudinal studies and for assessing the validity of follow-up data. Contact difficulty was examined among 96 substance abusers following substance abuse treatment. Interview completion rates at the 3-month and 6-month follow-ups were 93 and $97 \%$, respectively. The extent of contact efforts required to complete follow-up interviews varied substantially but tended to be greater at the 3-month follow-up than at the 6-month follow-up. Contact difficulty was related to reuse of substances at the 3-month and at the 6-month follow-ups with reusers requiring greater contact efforts than abstainers. None of the baseline individual and contextual variables examined significantly predicted level of contact effort at follow-ups. Attrition-related validity implications are discussed along with practical suggestions for planning tracking efforts.

KEY WORDS: substance abuse; tracking; treatment outcome; contact difficulty; relapse.

${ }^{1}$ This research was supported by the Michigan Department of Public Health, Center for Substance Abuse Services, the National Institute on Drug Abuse Grant DA05524, and the National Institute on Alcohol Abuse and Alcoholism Grant AA07477.

${ }^{2}$ All correspondence should be addressed to Maureen A. Walton, University of Michigan, Alcohol Research Center, 400 E. Eisenhower Parkway, Building 2, Suite A, Ann Arbor, Michigan 48108. 
A primary challenge facing researchers conducting substance abuse treatment outcome studies is successfully locating and assessing participants at follow-up assessments (Capaldi \& Patterson, 1987; Ribisl et al., 1996; Twitchell, Hertzog, Klein, \& Schuckit, 1992). Follow-up rates vary greatly by study with attrition rates ranging from less than $10 \%$ to more than $50 \%$ (Capaldi \& Patterson, 1987; Ribisl et al., 1996; Twitchell et al., 1992). Although statistical approaches to compensate for attrition are available (McArdle \& Hamagami, 1992), the most desirable approach is to reduce attrition by implementing tracking techniques that are demonstrated as effective (Ribisl et al., 1996; Twitchell et al., 1992). Because some attrition is inevitable in longitudinal follow-up studies, investigators have attempted to predict contact difficulty and to examine the nature of the possible bias that attrition might cause in the interpretation of study findings. This practice results from the knowledge that incomplete data can compromise the internal and external validity of a study, because those who were not assessed may have differed on the independent and dependent variables affecting the results and subsequent conclusions (Cook \& Campbell, 1979). For example, a popular assumption among clinicians and researchers is that substance abusers who are more difficult to contact or drop out of studies have relapsed. In addition, identification of individual and contextual characteristics that predict contact difficulty has tremendous utility for planning longitudinal studies. Descriptive data regarding project procedural successes and failures also can assist investigators in designing follow-up studies. This paper provides additional evidence regarding the relationship between contact difficulty and substance reuse and whether individual and contextual characteristics can be identified that predict contact difficulty.

\section{Contact Difficulty and Treatment Outcome}

Although researches and clinicians have frequently assumed that participants who are more difficult to contact following treatment have poorer outcome, few studies have systematically tested this assumption. Before reviewing studies that examine contact difficulty and substance abuse outcomes, a definition of contact difficulty is necessary to interpret the literature. Contact difficulty is generally defined as either the amount of time or the number of attempts required to locate a participant and complete a follow-up assessment. Three studies have found a relationship between contact difficulty and treatment outcome. For instance, Bale, Arnoldussen, and Quittner (1984) studied male substance abusers and found at 6-month follow-up that the length of time required to contact a participant was related to drinking, but in a nonlinear fashion. Participants 
requiring less time to complete a questionnaire were more likely to be abstinent or drinking daily. Participants drinking moderately required significantly greater time to contact. Polich, Armor, and Braiker (1980) found at 4-year follow-up that the time to contact alcoholic males was related to treatment outcome (in terms of drinking behavior, symptoms, and consequences) until a $60-70 \%$ response rate was reached, after which no relationship between effort and outcome was found. The authors concluded that locating the remaming $30 \%$ of the subjects is probably not worth the effort. In the only study to include women in their sample, Moos and Bliss (1978) found at a 5-month follow-up that the number of attempts required to contact alcoholics was related to alcohol consumption.

In contrast, two studies concluded that contact difficulty is not related to treatment outcome. Nordstrom and Berglund (1986) did not find a difference in return to problem drinking based on contact difficulty among male alcoholics. LaPorte, McLellan, Erdlen, and Parent (1981) examined contact difficulty at 6-month follow-up among male substance abusers. When conducting the analyses however, the authors used pretreatment scores as covariates in analyses examining contact difficulty and several outcome areas. They did not find a relationship between the number of location contacts and treatment outcome (including alcohol and drug use, employment, family/social, and psychological problems). The rationale for controlling for pretreatment functioning was not clear making the interpretation of this study's findings difficult. Regardless of whether pretreatment differences account for the relationship between contact difficulty and outcome, if contact difficulty is related to treatment outcome then the validity of the data could have been compromised had not extreme contact efforts been made. Nonetheless, the authors concluded that there was no difference in treatment outcome between a $60 \%$ follow-up rate and a $90 \%$ follow-up suggesting that studies with follow-up rates of $65-85 \%$ may be valid.

The issue of whether contact difficulty is related to treatment outcome requires further study. Of the five studies to systematically examine contact difficulty and outcome, only one included women in their sample. The results of these studies are also contradictory with some concluding that follow-up efforts of $70 \%$ are adequate and other concluding that they may be biased. Definitions of treatment outcome have varied in these studies; however, how these varying outcome definitions influenced these findings is not clear.

\section{Predictors of Contact Difficulty}

A related area of research has examined predictors of contact difficulty. Examination of characteristics that predict contact difficulty could 
assist investigators by identifying participants likely to require extensive efforts so that additional follow-up measures can be planned. Most of these studies examine individual characteristics, typically either demographic factors or baseline psychosocial functioning factors. Studies attempting to predict contact difficulty using individual characteristics have found inconsistent results. Regarding demographic factors, Bale et al. (1984) found that age and ethnicity were related to contact difficulty in univariate analyses, although the direction of this relationship was not specified, but not in multivariate analyses with other functioning indices. Moos and Bliss (1978) found that substance abusers who are more difficult to contact following treatment tended to be younger, not married, and have less income and education than those that were more easily contacted.

Similarly, studies find few consistent pretreatment functioning predictors of contact difficulty. LaPorte et al. (1981) found substance abusers' pretreatment alcohol, social, legal, and psychological functioning did not differentiate participants grouped by degree of contact difficulty. Contact difficulty was related to pretreatment amphetamine use, money spent on drugs, drug use severity, and days of psychological problems. Bale et al. (1984) found contact time was predicted by having criminal history, spending longer time in treatment, and having a previous treatment history.

Pretreatment functioning distinguishes dropouts from those completing follow-up assessments, in contrast to results for contact difficulty. Vanicelli, Pfau, and Ryback (1976) found dropouts were rated at baseline by staff as lower functioning. Nordstrom and Berglund (1986) found those lost at follow-up had greater baseline personality disturbances. Sobell, Sobell, and Maisto (1984) found alcoholics lost at follow-up had poorer baseline functioning than those found. Interestingly, those who refused to be interviewed had initial functioning equivalent to those interviewed. Thus, it may be that participants who are dropouts because they are not located introduce more systematic bias because they have poorer functioning than participants who are drop-outs because they refused to be interviewed at follow-up. Consistent individual level predictors of contact difficulty have not been identified; additional research focusing on predictors of contact difficulty could assist investigators in planning follow-up studies.

Although individual characteristics may be important, these studies ignore other factors that may influence contact difficulty such as contextual characteristics. Contextual characteristics that could be examined include variables such as social network factors, exposure to substances, and relapse risk of the various settings in which participants' interact. Few studies have examined contextual level factors as determinants of contact difficulty. Two studies found that residential instability was related to greater contact difficulty (Bale et al., 1984; Moos \& Bliss, 1978). It is unclear from these data 
whether other contextual factors might be related to contact difficulty and requires further study.

Finally, contact difficulty may be influenced by project factors. For example, the ease of follow-up is affected by the extent of location information gathered at baseline, financial constraints that limit the amount of time spent locating participants, failure to ensure participants' confidentiality and establish rapport, assessments occurring at inconvenient times or locations, or lack of adequate reimbursements. Descriptive information regarding the impact of these project factors is also very useful in planning longitudinal studies.

This paper examines whether contact difficulty reflects substance reuse and individual and contextual characteristics that may be related to contact difficulty. In addition, this paper describes the contact efforts used by this project, so that recommendations can be offered to assist investigators in planning longitudinal studies.

\section{METHOD}

\section{Design and Procedures}

A quasi-experimental longitudinal design was used to assess the impact of a relapse prevention program following treatment completion. The first 58 participants recruited were assigned to attend a 10-week contextual based relapse prevention program. The following 38 participants recruited were assigned to a no-additional treatment comparison group. Attempts were made to interview participants 3- and 6-months later. This paper reports on the follow-up efforts; information regarding the relapse prevention groups is beyond the scope of this paper (see Reischl, Reischl, \& Ramanathan, 1994; Walton, Ramanathan, Walton, \& Hguyen, 1992).

Participants were recruited using a variety of methods including treatment counselor referral, flyers posted at Alano Clubs, and presentations made at treatment centers. During the initial contact, interested participants signed a "consent to be contacted" form and indicated when they would complete treatment. Community interviewers contacted participants to arrange the pretest after they completed treatment. At the pretest, participants signed an informed consent and completed several questionnaires (not reported on here). Participants were compensated for their time to the amount of $\$ 10$ for the pretest interview, $\$ 15$ for the 3-month follow-up interview, and $\$ 20$ for the 6-month follow-up interview.

Ninety-six persons treated in the last 6-months for alcoholism and/or drug abuse in an inpatient $(n=35)$, outpatient $(n=37)$, or residential 
$(n=24)$ treatment program participated in the study. Although unintended, a greater percentage the outpatient participants $(73 \%)$ and inpatient participants $(63 \%)$ were assigned to the control group than participants from residential (38\%) programs, $\chi^{2}(2)=7.80 p<.05$. The relapse prevention and control groups did not differ on any demographic characteristic including age, gender, marital status, income, or baseline alcohol or drug severity. Treatment programs were located in mid-Michigan area in suburban settings located in lower middle class neighborhoods. Persons currently on methadone maintenance were excluded from participating.

The majority of the participants were male (79\%), White (70\%), and not married (74\%). The average age was 35 years, but ranged from 20 to 58 years. Most participants were employed full-time $(66 \%)$. Half of the participants $(51 \%)$ had a high school diploma or equivalent (19\% did not have a diploma and $30 \%$ had at least some college). The average monthly income was $\$ 1,084$ (the median was $\$ 770$ ) but ranged from $\$ 0$ to $\$ 10,000$.

Most participants identified themselves as primarily alcoholic (66\%); the other participants (34\%) indicated that their primary problem was with another drug. Few participants (5\%) had been in substance abuse treatment only once; $66 \%$ had been treated two or three times and $29 \%$ had been treated more than three times. Participants' abstinence periods varied: $64 \%$ had less than 6 months of sobriety; 29\% had 6 to 12 months of sobriety; $7 \%$ had 13 to 16 months of sobriety. The participants in this study were similar to statewide treatment admissions. In 1991, 62\% of statewide admissions were for alcohol problems (Contextual Epidemiology Work Group, 1991). The majority of statewide admissions were male (68\%), and between ages 26 to $35(57 \%)$, and not married (76\%). In contrast to this sample, only $21 \%$ of statewide admissions were employed full-time and $45 \%$ were White; these differences are not surprising considering the city of Detroit is included in the statewide admissions.

\section{Interviewer Training}

Four undergraduate students, who were graduate school bound, were hired as community interviewers. They received 20 hours of classroom training on confidentiality, interviewing and tracking methodology, and safety procedures. All interviewers signed a confidentiality statement. The evaluation coordinator required staff to pass a mock interview "test" and observed staff conducting pilot interviews in order to ensure the quality of the data and the competence of the staff. In cases of emergency, the evaluation coordinator also conducted interviews. Regarding project safety procedures, interviewers conducting assessments in participants' homes were 
instructed to call the project when they arrived and before leaving; this served to notify the project of their location in addition to showing the participant someone knew the interviewer's whereabouts. Female interviewers were instructed to have another interviewer accompany them during home interviews, especially during evening hours or when they knew the participant lived alone. The evaluation coordinator monitored these tracking efforts.

\section{Tracking Procedures}

A variety of procedures were used to locate participants at follow-ups (Table I). At the baseline interview, participants provided the names, addresses, and phone numbers of at least three persons who would know their whereabouts and signed release statements allowing project staff to contact these persons. They also provided social security numbers and driv-

Table I. Tracking Techniques Used to Locate and Interview Participants at Follow-Ups

\begin{tabular}{|c|c|}
\hline Tracking strategy & Examples \\
\hline Participant information & $\begin{array}{l}\text { Name, home address, home phone, work address, and work } \\
\text { phone }\end{array}$ \\
\hline Collected & $\begin{array}{l}\text { Three significant others' names, relationship type, home } \\
\text { address, home phone, work phone } \\
\text { Social security number and drivers license number } \\
\text { Signed release of information allowing staff to contact } \\
\text { significant others and agencies for current address and } \\
\text { phone number }\end{array}$ \\
\hline Tracking procedures & $\begin{array}{l}\text { Business cards and stationery wih generic project logo, } \\
\text { telephone number (collect calls accepted), date of next } \\
\text { interview, and payment } \\
\text { Letter sent } 4 \text { weeks before the interview date. "Forwarding } \\
\text { address correction requested" printed on all correspondence } \\
\text { Cases assigned to interviewers two weeks vefore due to date } \\
\text { If location difficulties, repeated phone calls and letters to } \\
\text { all known addresses. Last known address visit and talk } \\
\text { with neighors. Agency checks (e.g., Department of Public } \\
\text { Health, Secretary of State, unemployment office) } \\
\text { If compliance difficulties, home visits to leave business } \\
\text { cards. Continue efforts until end of project or participant } \\
\text { refusal }\end{array}$ \\
\hline Participant incentives & $\begin{array}{l}\text { Payment for interview } \\
\text { Interview conducted at a location of the participant's choice } \\
\text { Refreshments provided } \\
\text { Confidentiality assured }\end{array}$ \\
\hline Interviewer incentives & $\begin{array}{l}\text { Hourly payment mileage payment, bonus payment for } \\
\text { contact efforts } \\
\text { Regular brain storming meetings }\end{array}$ \\
\hline
\end{tabular}


ers license numbers. Participants were given a card containing the project logo, contextual interviewers' name, project office phone number, next interview date, and payment. Since many participants were called during weekends and evenings, interviewers sometimes left their home numbers. Collect calls were accepted at the project office and the interviewers' homes. On all stationery and business cards, the project used a generic name (not related to substance use) to protect participant confidentiality. Because most participants' neighborhoods were lower middle class suburban settings, location procedures did not appear to vary systematically because of setting.

Locating Participants. Approximately 4 weeks before the scheduled follow-up date, a letter was sent to participants informing them of their upcoming interview and interview payment. "Forwarding address correction requested" was written on the envelope so that the post office would notify the project if a letter had been forwarded to a new address. Cases were assigned to interviewers 2 weeks before the scheduled date. Interviewers were expected to locate and interview participants within 2 weeks following the scheduled interview date; thus, interviewers had 4 weeks to complete each interview. Expectations were made that interviewers repeatedly call contact phone numbers until they reached the participant or someone who had information on the participant's whereabouts. If they could not reach the person within a week, additional letters were sent. If the participant did not have a phone at home or work, the interviewer went to their house usually early in the morning or during early evening hours (e.g., around dinnertime). If the participant was not home, interviewers either left a friendly, handwritten note asking the person to contact the project or waited for them to return. If an interviewer was not sure if a person lived at an address, a letter was sent by certified mail, return receipt requested. If the participant no longer lived at their last known address and no new address was known, interviewers would ask neighbors if they knew the person's current address. The Department of Public Health death records were checked for persons who could not be located. These contact efforts continued until after the 6-month interview was due.

Participant Compliance. All interviews were conducted at a location chosen by the participant including homes, work, restaurants, and Alano Clubs. Alano Clubs were particularly a good place to interview participants because interviewers felt safe at these locations and many participants would attend a meeting after the interview. At restaurants and Alano Clubs, interviewers paid for refreshments (e.g., coffee, soda). In all locations, interviewers made sure confidentiality was maintained such that no one could overhear the conversation. Another strategy used to enhance tracking was that the same interviewer that conducted the pretest interview, usually con- 
ducted the follow-up interviews. In this way, the participants appeared to feel comfortable with the person calling and sometimes looked forward to visiting with the interviewer again. Exceptions were made. For example, a male participant that made sexually suggestive comments to a female interviewer was reassigned to a male interviewer for future interviews.

Inteniewer Compliance. Several procedures were used to motivate community interviewers. First, interviewers were paid hourly for interviews and travel time and were reimbursed for mileage. They also were paid at an hourly rate for waiting outside participant's homes. In addition, for every 12 contact attempts made (by either phone or mail), interviewers were paid for 1 hour of work. The effectiveness of these monetary incentives was most likely enhanced for this project by the use of undergraduate student interviewers. Interviewers helped each other brainstorm case-finding alternative strategies at 1 -hour weekly meetings. Although interviewers were discouraged from trading cases, they frequently assisted each other in the location efforts. For example, one of the interviewers, who was a recovering alcoholic, sometimes obtained information about the location of a lost participant (that may have been that interviewers' case or another interviewers) during conversations around local Alano Clubs. This interviewer was careful not to violate confidentiality of the participants and did not indicate they were in the study.

\section{Measures}

Addiction Severity Index (ASI). In addition to demographic data, the ASI assesses pretreatment functioning in the following problem domains: medical, employment, social, drug and alcohol use, legal, and psychiatric. Composite scores computed from each domain indicate problem severity in each domain (McLellan et al., 1985a). These composite scores have been shown to be reliable and valid (McLellan et al., 1985b). For this paper, the alcohol and other drug composite scores were used to indicate pretreatment alcohol and other drug use problem severity.

Contact Logs. Tracking information was obtained from interviewers' contact logs. Community interviewers recorded all phone, mail, and in-person contact attempts, along with descriptive information regarding the outcome of these efforts. Letters sent by the evaluation coordinator approximately 4 weeks before the interview due date were not recorded on the contact logs. In addition, some participants required no phone, mail, or in-person contact efforts because they contacted the project to schedule their next interview. From these logs, the number of phone, mail, and in- 
person contacts was counted. The total number of contacts was determined by counting all contact attempts regardless of type.

Social Network. Social networks were delineated according to four areas: partner, family, friends, professionals, others (Norbeck, Lindsey, \& Carrieri, 1981). No limit was placed on the reporting of number of network members. A variety of questions were asked about each network member including whether the network member used alcohol or other drugs. A ratio of the number of nonusers in the network to the total number of network members was computed and used in analyses.

Setting Risk. Perceived risk for relapse was assessed using the Setting Risk Indicator (Walton et al., 1994). Participants reported the number of days during the past month that they had urges to use substances or felt at risk for relapse in their home, work, and contextual settings. To compensate for the skewed distributions, risk in each setting was recoded into ordinal responses: 0 indicated no risk days; 1 indicated $1-7$ risk days; 2 indicated more than 7 risk days. A setting risk variable was created by summing the three recoded risk responses (home, work, and contextual).

Exposure to Substances. Substance exposure was assessed also using the Setting Risk Indicator (Walton et al., 1994). Participants reported the number of days during the past month that they had been offered substances or in which someone used substances in their presence in their home, work, and contextual settings. Because participants' responses to these questions were skewed, responses were recoded into ordinal categories: 0 indicated no exposure days; 1 indicated 1-7 exposure days; 2 indicated more than 7 exposure days. The exposure to substances variable was created by summing the recoded home, work, and contextual responses.

Substance Reuse. The Timeline Calendar protocol was used to examine daily alcohol and drug consumption over the 3-month follow-up period (Sobell, Maisto, Sobell, \& Cooper, 1979); several studies have demonstrated the reliability and validity of this method (Sobell, Sobell, Leo, \& Caneilla, 1988). At the follow-up, $72 \%$ of the participants remained abstinent and $28 \%$ reused substances. Although relapse is clearly not a uniform phenomenon, because the number of days of substance use was highly skewed both by the number of abstainers and the large number of days in which some people relapsed, a dichotomous abstinence/reuse variable was used for outcome analyses.

\section{Data Analysis}

Contact difficulty at 3-month and 6-month follow-up was highly skewed which reflects the extreme efforts needed to contact a few partici- 
pants. Thus, descriptions of contact efforts made by the project are described using quartile cutoffs, box plots, and survival curves. To examine whether contact difficulty reflects substance reuse, total contact difficulty at the 3- and 6-month follow-ups was categorized by using approximately the third quartile where ten contacts served as the cutoff between low and high contact difficulty. The reason for categorizing these variables was because we thought it was theoretically more interesting to compare those requiring low and high contact efforts. Furthermore, because contact difficulty was highly skewed, it could not be appropriately analyzed without a statistical or categorical transformation. Similarly, when examining individual and contextual characteristics that are related to contact difficulty, logistic regression analyses were used where low or high contact difficulty served as the dependent variable.

\section{RESULTS}

\section{Contact Difficulty}

At the 3-month follow-up, 89 of 96 participants completed the interview, and at the 6-month follow-up, 93 of 96 participants completed the follow-up interview; thus the completion rates for the two follow-ups were 93 and $97 \%$, respectively. Of the 7 participants not interviewed at the 3month follow-up, 1 person withdrew their participation from the study, 2 were not located, and 4 missed appointments; thus the 3-month location rate was $98 \%$. Of the 3 persons who were not interviewed at the 6-month follow-up, 1 person withdrew their participation from the study, another person was in jail and refused to be interviewed, and the third person was not located; thus the 6-month location rate was $99 \%$.

Because of the number of contact attempts was positively skewed with the 25th quartile, median, 75th quartile, and range of phone, mail, in-person, and total contact attempts for the 3-month and 6-month interviews for participants that completed interviews are presented in Table II. The most frequently used contact effort was phone contacts, followed by in-person attempts, and mail attempts, respectively. The number of contact attempts of all types tended to be greater at the 3-month follow-up than at the 6month follow-up. At 3-months, phone contacts were significantly correlated with mail $(r=.33, p<.001)$ and in-person $(r=.45, p<.001)$ contacts. At 6 months, phone contacts were not significantly correlated with either mail $(r=-.06)$ or in-person $(r=.05)$ contacts. It may be that the reduced variance in mail and in-person variables at 6 months influenced this finding. Also evident from Table II, the intensity of total contact attempts made as 
Table II. Number of Contact Attempts at Follow-Ups ${ }^{a}$

\begin{tabular}{lcccccccccc}
\hline & \multicolumn{3}{c}{ 3-month follow-up } & & \multicolumn{3}{c}{ 6-month follow-up } \\
\cline { 2 - 4 } \cline { 8 - 10 } Contact type & $\begin{array}{c}\text { 25th } \\
\text { quartile }\end{array}$ & Median & $\begin{array}{c}\text { 75th } \\
\text { quartile }\end{array}$ & Range & & $\begin{array}{c}\text { 25th } \\
\text { quartile }\end{array}$ & Median & $\begin{array}{c}\text { 75th } \\
\text { quartile }\end{array}$ & Range \\
\hline Phone & 3 & 5 & 9 & $0-46$ & & 2 & 3 & 8 & $0-36$ \\
Mail & 0 & 0 & 1 & $0-3$ & & 0 & 0 & 1 & $0-2$ \\
In-person & 1 & 1 & 1 & $0-4$ & & 1 & 1 & 1 & $0-11$ \\
Total & 4 & 7 & 10 & $1-50$ & & 3 & 5 & 10 & $1-37$ \\
\hline
\end{tabular}

${ }^{a}$ Interview completion rate: $93 \%$ at 3 -months, $97 \%$ at 6 -months.

indicated by the median contact attempts (seven 3-month contacts and five 6-month contacts). Figure 1 shows a box plot of the total number of contact efforts made at 3- and 6-month follow-ups. As can be seen from Figure 1, the total number of contact attempts made was variable, ranging from 1 to 50 attempts at the 3-month follow-up and 1 to 37 attempts at the 6-month follow-up. Figure 2 shows the cumulative percentage of participants completing interviews for the total contact attempts made at 3-month and 6month interviews. As is evident from Figure 2 approximately $75 \%$ of participant interviews were completed within 10 attempts, with approximately $90 \%$ of participants completing interviews in 22 attempts at the 3month follow-up and 13 attempts at the 6-month follow-up.

\section{Contact Difficulty and Reuse}

To test whether contact difficulty reflected reuse such that failure to include participants requiring high contact efforts would compromise study findings, chi-square analyses were conducted between low and high contact groups and abstainers and reusers at 3 months and 6 months (Table III). Total number of 3-month contacts was significantly related to reuse status at 3 months, $\chi^{2}(1)=4.36, p<.05 ; 22.4 \%$ of the low contact effort group reused substances in comparison to $45.5 \%$ of the high contact effort group. At the 6-month follow-up, total contacts was significantly related to reuse status, $\chi^{2}(1)=5.05, p<.05 ; 32.4 \%$ of the low contact effort group reused substances in comparison to $61.1 \%$ of the high contact effort group.

\section{Individual and Contextual Characteristics and Contact Difficulty}

Several individual and contextual characteristics were hypothesized to indicate participants who would be more difficult to contact. Individual 

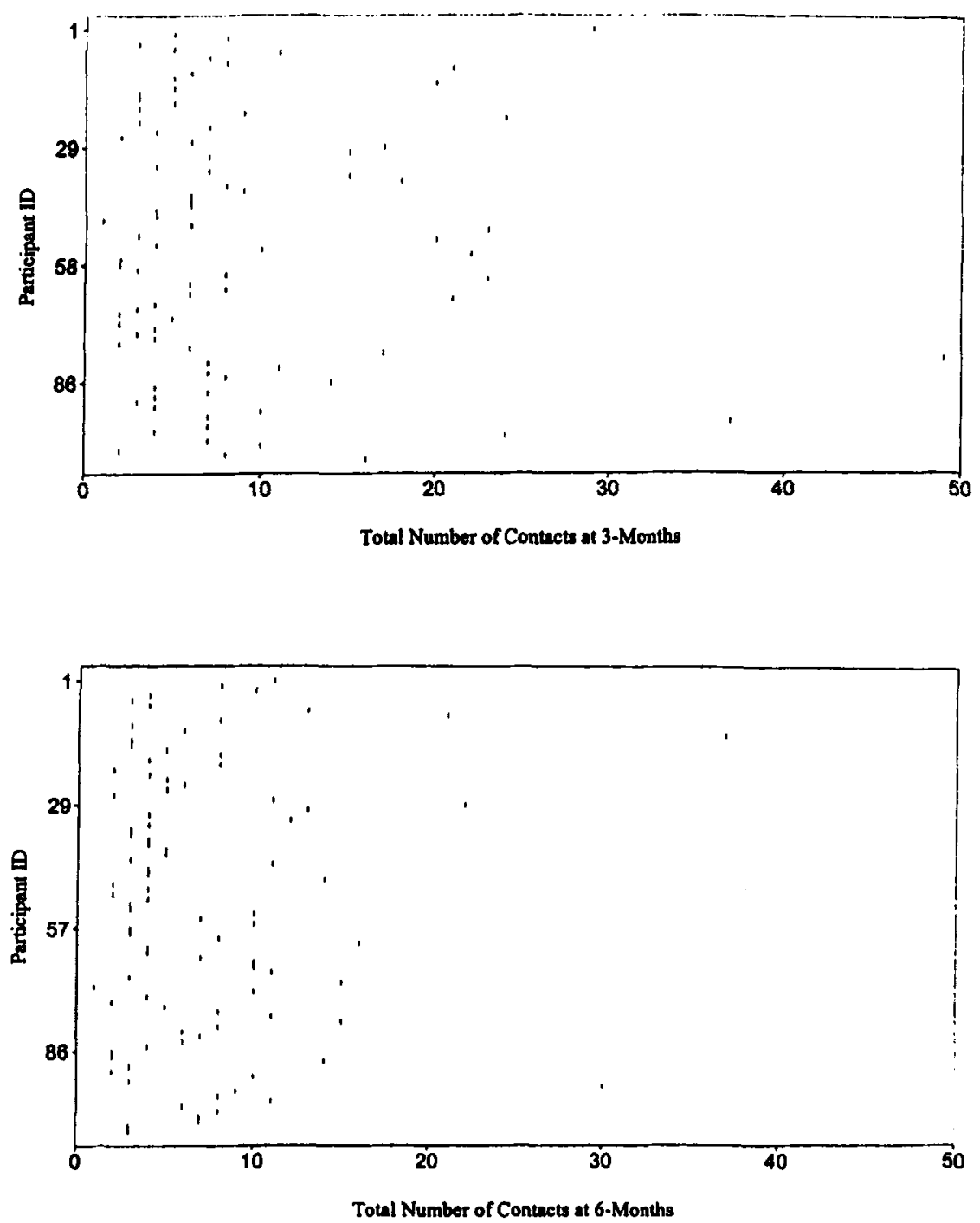

Fig. 1. Distribution of total contactsat 3 months and 6 months

variables examined included gender, marital status (married, not married), income $(<\$ 1,000$ per month, $>\$ 1,000$ per month), number of previous 

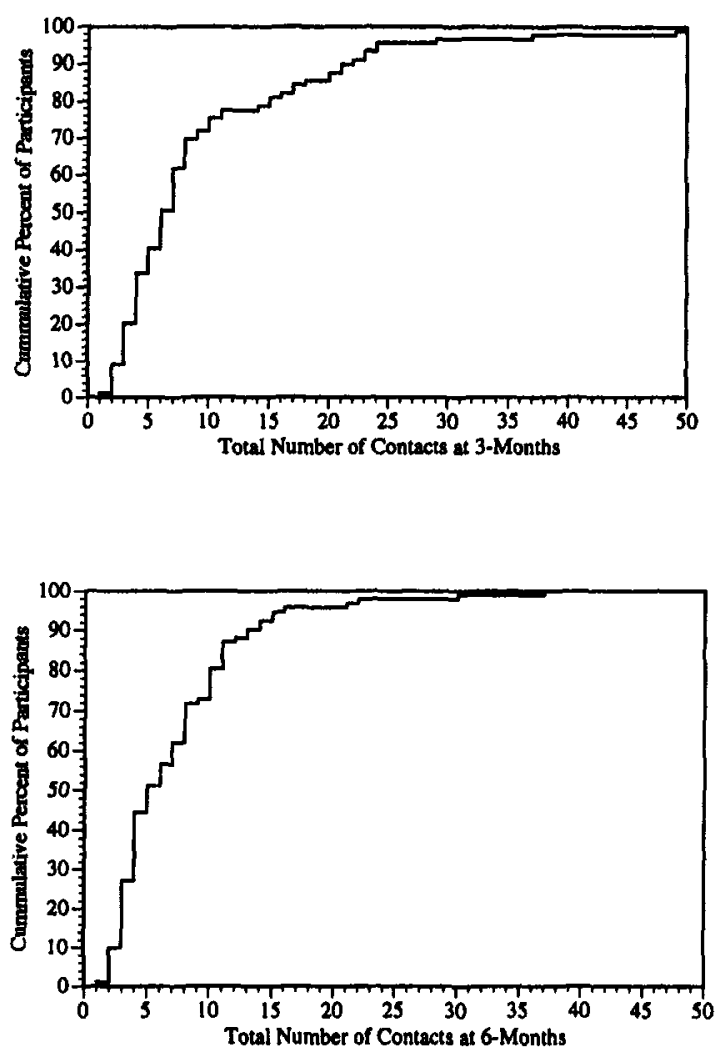

Fig. 2. Cummulative percentage of participants completing follow-up interviews based on the total number of contact attempts.

treatments $(0,1,2,3$ or more), drug of choice (alcohol or other drugs), and amount of sobriety time. Contextual variables examined included group assignment (relapse prevention or control), treatment site (outpatientyes/no, or, residential-yes/no), setting risk, setting exposure, and proportion of non-substance users in their network. None of these variables was correlated above .35 except for the dummy variables for treatment site (outpatient and residential) which were correlated -0.46 , and drug of choice was correlated .36 with outpatient treatment.

Logistic regression analyses were conducted to determine if the relationship between contact difficulty and reuse at 3 months and 6 months was a function of individual and contextual factors. Because of power concerns due to the sample size and the number of variables to be included, separate hierarchical logistic regression analyses were conducted for indi- 
Table III. Percentage of Participants in Low and High Contact Effort Groups by Reuse Status at Follow-Ups

\begin{tabular}{lcccccc}
\hline & \multicolumn{2}{c}{ 3-month follow-up } & & \multicolumn{2}{c}{ 6-month follow-up } \\
\cline { 2 - 3 } \cline { 6 - 7 } Contact difficulty group & \%Abstainers & \%Reusers & & \%Abstainers & \%Reusers \\
\hline Low contact effort & 77.6 & 22.4 & & 67.6 & 32.4 \\
High contact effort & 54.5 & 45.5 & & 38.9 & 61.1 \\
\hline
\end{tabular}

vidual and contextual factors. (Assuming a moderate effect size, $p<.05$, and a desired power of $0.80,91$ participants are needed when including five independent variables and 97 participants are needed when including six independent variables in a regression. Cohen, 1992.) In both cases, low or high contact difficulty served as the dependent variable (Tables IV and V). None of the individual or contextual characteristics examined significantly predicted either 3- or 6-month follow-up status. There was a trend at 3-months for individual baseline characteristics to predict contact difficulty, with the beta for income approaching significance in the expected direction.

Table VI shows descriptive data for the independent variables examined above according to reuse status. When univariate analyses were conducted, marital status significantly distinguished low and high contact difficulty, $\chi^{2}(1)=4.03, p<.05$, at 3 months, with married participants more likely to be in the low contact difficulty group (31.9\%) than in the high contact group (11.5\%). Univariate tests showed that none of the other independent variables were significantly related to contact difficulty groups at 3 months or 6 -months.

\section{DISCUSSION}

Results from this study show that contact difficulty is associated with reuse, where substance abusers requiring intense contact efforts were more likely to have relapsed. This issue is important because it suggests that the additional cost needed to contact some participants is necessary to ensure the validity of follow-up data. In addition, results suggest that is it difficult to identify at baseline individual and contextual characteristics that might identify participants who are difficult to contact at follow-up. Thus, investigators planning longitudinal studies should implement comprehensive tracking efforts, such as those used in this study, for all participants. 
Table IV. Logistic Regression Analyses for Individual Factors Predicting Follow-Up Contact Efforts

\begin{tabular}{lcccccc}
\hline & \multicolumn{2}{c}{ 3-month follow-up } & & & \multicolumn{2}{c}{ 6-month follow-up } \\
\cline { 2 - 3 } \cline { 6 - 7 } \multicolumn{1}{c}{ Variable } & $\beta$ & Wald & & $\beta$ & Wald \\
\hline Gender & .61 & 2.58 & & -.12 & 0.10 \\
Marital Status & -.56 & 2.46 & & -.08 & 0.06 \\
Income & .55 & $3.02^{c}$ & & .11 & 0.11 \\
\# of Previous Treatments & -.06 & 0.10 & & -.24 & 1.08 \\
Sobriety Time & -.00 & 0.00 & & -.04 & 0.35 \\
Drug of Choice & -.07 & 0.07 & & -.19 & 0.45
\end{tabular}

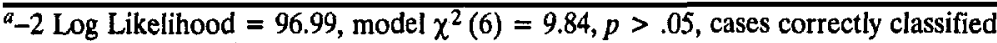
$=74.4 \%(n=90)$.

${ }^{b}-2$ Log Likelihood $=87.29$, model $\chi^{2}(6)=2.90, p>.05$, cases correctly classified $=79.6 \%(n=88)$.

${ }^{c} p<.10$.

Table V. Logistic Regression Analyses for Contextual Factors Predicting Follow-Up Contact Efforts

\begin{tabular}{|c|c|c|c|c|}
\hline \multirow[b]{2}{*}{ Variable } & \multicolumn{2}{|c|}{ 3-month follow-up ${ }^{a}$} & \multicolumn{2}{|c|}{ 6-month follow-up } \\
\hline & $\boldsymbol{\beta}$ & Wald & $\beta$ & Wald \\
\hline Group assignment & -.15 & 0.34 & -.12 & 0.18 \\
\hline No. of nonusers & -.11 & 2.10 & -.05 & 0.60 \\
\hline Residential treatment & -.39 & 1.46 & -.04 & 0.01 \\
\hline Outpatient treatment & -.46 & 2.18 & -.45 & 1.93 \\
\hline Setting risk & .24 & 2.66 & -.15 & 0.64 \\
\hline Exposure & -.28 & 1.75 & -.09 & 0.15 \\
\hline
\end{tabular}

Tracking procedures used by this project were successful in locating and assessing over $90 \%$ of the original sample at 3-month and 6-month follow-ups. Contact efforts varied by type of contact attempt and by follow-up. Phone contacts were the most frequently used method of contacting participants and, as such, had the most variability in the number of calls made. The interview completion rate obtained in this study required intensive efforts on the part of the interviewers. Although the median number of contact attempts made was 7 at the 3-month follow-up and 5 at the 6-month follow-up, as many as $\mathbf{5 0}$ attempts were made at the 3-month follow-up 37 at the 6-month follow-up. More contact attempts tended to be made at the 3-month follow-up than the 6-month follow-up because (a) 
Table VI. Descriptive Data for Individual and Contextual Characteristics Based on Reuse

\begin{tabular}{|c|c|c|c|c|}
\hline \multicolumn{5}{|c|}{ Status } \\
\hline \multirow[b]{2}{*}{ Variable } & \multicolumn{2}{|c|}{ 3-month follow-up } & \multicolumn{2}{|c|}{ 6-month follow-up } \\
\hline & Abstainers & Reusers & Abstainers & Reusers \\
\hline \multicolumn{5}{|l|}{ Individual characteristics } \\
\hline Gender ( $\%$ male $)$ & 78.3 & 84.6 & 79.7 & 78.9 \\
\hline Marital status (\% married) & 31.9 & 11.5 & 27.0 & 21.1 \\
\hline Income $(\%<\$ 1,000 /$ month $)$ & 62.3 & 80.8 & 66.6 & 68.4 \\
\hline No. of treatments $(M \pm S D)$ & $2.65 \pm 3.11$ & $2.15 \pm 1.71$ & $2.70 \pm 3.03$ & $1.95 \pm 1.75$ \\
\hline Sobriety time $(M \pm S D)$ & $5.06 \pm 4.34$ & $4.59 \pm 3.82$ & $5.14 \pm 4.08$ & $4.54 \pm 4.67$ \\
\hline Drug of choice (alcohol \%) & 71.0 & 65.4 & 71.6 & 57.9 \\
\hline \multicolumn{5}{|l|}{ Contextual characteristics } \\
\hline Group assignment (\% control) & 62.3 & 57.7 & 60.8 & 57.9 \\
\hline No. of nonusers $(M \pm S D)$ & $7.10 \pm 4.76$ & $5.69 \pm 3.26$ & $6.77 \pm 4.45$ & $6.10 \pm 4.47$ \\
\hline Residential treatment (\%) & 23.2 & 30.8 & 27.0 & 21.1 \\
\hline Outpatient treatment (\%) & 36.2 & 46.2 & 36.5 & 52.6 \\
\hline Setting risk $(M \pm S D)$ & $1.58 \pm 2.19$ & $2.19 \pm 2.04$ & $1.82 \pm 1.67$ & $1.53 \pm 1.54$ \\
\hline Exposure $(M \pm S D)$ & $1.29 \pm 1.37$ & $1.23 \pm 1.14$ & $1.28 \pm 1.36$ & $1.26 \pm 1.20$ \\
\hline
\end{tabular}

participants appeared to be most mobile, or least stable, in their residences immediately following treatment discharge, and (b) once participants were located for the first follow-up interview, they were more likely to stay in contact with the project and be located and compliant with the second follow-up. This finding is consistent with findings from substance abuse prevention programs that attrition is greatest at the first follow-up (Hansen, Tobler, \& Graham, 1990). Thus, researchers should allow for intensive tracking efforts during initial follow-ups.

A variety of tracking strategies were used in this study to produce the successful follow-up interview completion rate. In our experience, locating participants for follow-up interviews is not difficult given appropriate participant information and releases were obtained at baseline, and that sufficient project resources are available to repeatedly use the tracking procedures described. We found that the more difficult task was scheduling the appointment once the participants whereabouts were known and compliance with interview appointments. One factor that influenced the interviewers' ability to schedule the appointments were that interviewers' persistence. Our interviewers quickly became frustrated by participants who were seldom home. Interviewers also noted that participants' who were in the middle of a drinking or drug-using binge would be under the influence when the interviewer arrived at their homes. Repeated appointments would be made and broken until the participant would be sober for the interview; sometimes this process took several weeks. It is for that reason that an 
additional monetary incentive was provided to interviewers for contact attempts made. In addition, brainstorming meetings were a place where staff members could support each other in their frustration which help build morale. Next, we found that compliance with interviewer appointments was best when the interview went to the participants' home. Participants were less likely to attend appointments at restaurants or other locations. Interviewers also noted that participants were frequently not willing to return to the treatment center for interviews. This was typically because they had relapsed, or that they had a financial dispute with the treatment agency. Investigators planning longitudinal studies should be prepared to address interviewer burnout, and to incorporate adequate staff, time, and resources for in-home interviewing.

An important concern in recommending home interviewing is safety issues. Safety procedures used by this project were that interviewers immediately call the project office upon arriving at their appointment and before leaving. This served to notify the participant that someone on the project knew where the staff member was. If the interview was conducted after hours, the staff member called one of the other interviewers to confirm their location. Female interviewers interviewing male respondents often had another staff member accompany them on an interview. Cases in which the participant made sexually suggestive comments to interviewers were reassigned to a same-sex interviewer. Most home interviews were conducted during daytime hours, or when participants' family members were home. Finally, staff were encouraged to immediately reschedule any interview in which they felt unsafe.

Conclusions regarding the validity of follow-up data, and the justification of extraordinary efforts, vary by study according to whether a relationship between contact difficulty and substance abuse treatment outcome is found. In this study, the percentage of reusers at 3-month and 6-month follow-ups in the high contact effort group was significantly greater than the percentage of reusers in the low contact effort group. Considering that the high contact effort group consisted of approximately one quarter of the sample, this finding suggests that results regarding treatment outcome could be compromised had not these extensive efforts been taken. Disproportionally missing some of the relapsers could compromise the internal validity of the study as well as the generalizability of the findings. This finding is consistent with results of some studies (Bale et al., 1984; Moos $\&$ Bliss, 1978) but is in conflict with findings from others studies (LaPorte et al., 1981; Polich et al., 1980). Discordant findings, as compared to that of Polich et al., may be explained by the length of the follow-up period examined (4 years in the former vs 6-months in this study). LaPorte et al. differed in their analysis approach as they did not examine the direct effects 
of contact difficulty and reuse, but instead controlled for pretreatment characteristics, reporting a null finding. Both of these studies included only males in their samples; although, no gender effects were observed in this study. Finally, the conflicting results may be a function of the strength of the relationship between contact difficulty and reuse. If in fact the relationship between contact difficulty and relapse is modest, issues of sample size and power may explain why some study findings reach significance and others do not.

Regardless, results from this study suggest contact difficulty may reflect reuse with relapsed participants being more difficult to find. Participants who have relapsed may be more difficult to locate and interview because of both individual and contextual factors. Persons who are using may be less compliant with interviews because substance use becomes their main priority. From a contextual standpoint, they may be more difficult to locate as they become less connected to the contact names they provided (e.g., family members) and more connected to persons involved in the drug subculture. Because over $90 \%$ of the participants were located at the follow-ups, analyses further examining validity issues related to attrition, by comparing those interviewed with those not interviewed, were not possible.

Data from this study suggest that it is difficult to identify at baseline who will be challenging to locate and interview at follow-up. Results do not confirm other findings from the literature that participants of lower income required greater contact efforts. From our experience, however, a socioeconomic factor that appears to make location more difficult and was not systematically measured in this study was whether the participant had a telephone. In an univariate analysis, marital status was related to contact difficulty with married participants requiring less effort at the 3-month follow-up. This finding for marital status is consistent with the literature (Bale et al., 1984; Moos \& Bliss, 1978) and suggests that researchers may want to collect additional contact information for persons who are not married, or they may want to begin tracking these people earlier. In our experience, unmarried participants were more difficult to interview because they moved residences more often than married participants. Drug of choice did not predict follow-up contact difficulty which is consistent with other studies where the severity of alcohol or other drug problems at baseline did not predict follow-up contact difficulty (Bale et al., 1984; LaPorte et al., 1981).

Contextual factors such as the proportion of non-drug-using members in the participant's social network, exposure to substances, and relapse risk in social settings were not related to contact difficulty. Another contextual factor that may be related to location that was not examined systematically in this study was stability of residence. Until further empirical data exist regarding factors influencing contact difficulty, the best recommendation 
for conducting longitudinal studies is to utilize the tracking procedures outlined in this study and others (Ribisl et al., 1996; Twitchell et al., 1992) for all participants. Project characteristics that might influence follow-up location, such as interviewer resourcefulness, could not be examined in this study. As stated above, in our experience the most powerful indicator of follow-up interview location is project resources. Even with the best planning, studies without staff to make home visits and spend enormous amounts of time to locate a few individuals have less success.

Care should be taken when generalizing the results of this study due to the brief follow-up period and to possible selectivity biases present when requiring participants to complete treatment and volunteer for a research study. No information was available regarding the number or characteristics of persons in treatment not participating; however, the sample appears to be similar to statewide treatment admissions on demographic characteristics. Nonetheless, replication is warranted. The sample selection procedures used in this study may explain why the majority of participants were abstinent over the 6-month follow-up period. Because of the limited number of participants who reused any substance, this study compared participants who reused substances (regardless of the extent of substance use) to abstainers. Although relapse is clearly not a uniform phenomenon, the findings contribute to the understanding of how abstainers and reusers differ in tracking efforts. The detailed information collected regarding the tracking efforts, the high follow-up interview completion rate, and the examination of individual and contextual factors are strengths of this study. Results suggest there is some evidence that validity is compromised because participants requiring extreme contact efforts are more likely to have reused substances. Future studies examining contact difficulty and substance abuse treatment outcome should consider assessing individual, contextual, and project factors in order to better plan follow-up efforts.

\section{REFERENCES}

Bale, R. N., Arnoldussen, B. H., \& Quittner, A. M. (1984). Follow-up difficulty with substance abusers: Predictions of time to locate and relationship to outcome. International Journal of the Addictions, 19, 885-902.

Capaldi, D., \& Patterson, G. R. (1987). An approach to the problem of recruitment and retention rates for longitudinal research. Behavioral Assessment, 9, 169-178.

Cohen, J. (1992). A power primer. Psychological Bulletin, 112, 155-159.

Contextual Epidemiology Work Group. (1991, June). Epidemiological trends in drug abuse. Rockville, MD: National Institute on Drug Abuse.

Cook, T. D., \& Campbell, D. T. (1979). The conduct of randomized experiments. In Quasi-Experimentation: Design \& analysis issues for field settings (pp. 341-386). Boston: Houghton Mifflin. 
Graham, J. W., \& Donaldson, S. I. (1993). Evaluating interventions with differential attrition: The importance of nonresponse mechanisms and the use of follow-up data. Journal of Applied Psychology, 78, 119-128.

Hansen, W. B., Tobler, N. S., \& Graham, J. W. (1990). Attrition in substance abuse prevention research: A meta-analysis of 85 longitudinally followed cohorts. Evaluation Review, 14, 677-685.

LaPorte, D. J., McLellan, A. T., Erdlen, F. R., \& Parente, R. J. (1981). Treatment outcome as a function of follow-up difficulty in substance abusers. Journal of Consulting and Clinical Psychology, 49, 112-119.

McArdle, J., \& Hamagami, F. (1992). Modeling incomplete longitudinal and cross sectional data using latent growth structural models. Experimental Aging Research (Quarterly Topics in Research in Aging, Special Issue), 18(3), 145-166.

McLellan, A. T., et al. (1985a). Guide to the Addiction Severity Index (DHHS Publication No. ADM 85-1419). Rockville, MD: National Institute on Drug Abuse.

McLellan, A. T., Luborsky, L., Cacciola, J., Griffith, J., Evans, F., Barr, H. L., \& O'Brien, C. P. (1985b). New data from the addiction severity index. Joumal of Nervous and Mental Disease, $173,412-423$.

Nordstrom, G., \& Berglund, M. (1986). Successfully adjusted alcoholics lost to long-term follow-up. A prospective study. Drig and Alcohol Dependence, 18, 11-22.

Norbeck, J., Lindsey, A., \& Carrieri, V. (1981). The development of an instrument to measure social support. Nursing Research, 30, 264-269.

Polich, J. M., Armor, D. J., \& Braiker, H. B. (1980). The course of alcoholism: Four years after treatment (Report R-2433-NIAAA). Santa Monica, CA: Rand.

Reischl, T. M., Ramanathan, C.S., Walton, M.A., \& Hguyen, H. (1992). Michigan addiction relapse prevention project: Final Report. Detroit; Michigan Department of Public Health Center for Substance Abuse Services.

Ribisl, K. M., Walton, M. A., Mowbray, C. T., Luke, D. A., Davidson, W. S., \& Boots, B. J. (in press). Tracking hard-to-locate participants in longitudinal research: Review and recommendations. Evaluation and Program Planning.

Sobell, L. C., Maisto, S. A., Sobell, M. B., \& Cooper, A. M. (1979). Reliability of alcohol abusers' self-reports of drinking behavior. Behaviour Research and Therapy, 17, 157-160.

Sobell, L. C., Sobell, M. B., Leo, G. I., \& Caneilla, A. (1988). Reliability of a timeline method: Assessing normal drinkers' reports of recent drinking and a comparative evaluation across several populations. British Journal of Addiction, 83, 393-402.

Sobell, L. C., Sobell, M. B., Maisto, S. A. (1984). Follow-up attrition in alcoholism treatment studies: Is "no news" bad news, good news, or no news? Drug and Alcohol Dependence, $13,1-7$.

Twitchell, G. R., Hertzog, C. A., Klein, J. L., \& Schuckit, M. A. (1992). The anatomy of a follow-up. British Joumal of Addiction, 87, 1327-1333.

Vanicelli, M., Pfau, B., \& Ryback, R. S. (1976). Data attrition in follow-up studies of alcoholics. Journal of Studies on Alcohol, 37, 1325-1329.

Walton, M. A., Reischl, T. M., Ramanathan, C. S. (1994). Social settings and addiction relapse. Journal of Substance Abuse, 7, 223-233. 